\title{
Legged Robots
}

Claudio Semini and Pierre-Brice Wieber

\section{Author Info}

\section{Claudio Semini}

Dynamic Legged Systems (DLS) lab

Istituto Italiano di Tecnologia (IIT)

Genova, Italy

claudio.semini@iit.it

\section{Pierre-Brice Wieber}

INRIA, Grenoble - Rhône-Alpes Research Centre

Montbonnot-Saint-Martin, France

pierre-brice.wieber@inria.fr

\section{Synonyms}

Walking robots, running robots, jumping robots, climbing robots, limbed systems, walking machines, legged vehicles.

\section{Definition}

Legged Robots use legs to move from one place to another. Legs provide an active suspension [56], so the motion of the main body of the robot can be largely decoupled from the terrain profile. With each step, a leg is temporarily lifted off the ground, so that discontinuous terrain can be overcome as well, allowing locomotion in places out of reach otherwise. 
Legs are usually articulated rigid bodies, assumed to contact the environment only with their end effector. In most cases, this contact is unilateral, meaning that the robot can push but not pull on contact surfaces. In some cases, grasping, suction cups, magnets, adhesive materials or miniature spine arrays can provide additional grip [27, 33, 61].

Adapting wheeled vehicles to rough terrain has led in some cases to implant wheels on legs, with any combination of passive or active wheels, passive or active legs, combining the flexibility of articulated legs on rough terrain with the efficiency of wheels on flat terrain $[16,30]$. On steep slopes, legged robots can also use rappelling to avoid tumbling down [5].

\section{Mechanical Design}

Hundreds if not thousands of legged robots have been designed in the past decades. Figure 1 shows a very small selection of well-known legged robots, ranging from monohoppers and bipeds to quadrupeds and hexapods.

Legged robots are composed of a central body (also called trunk or torso) with legs attached to it. Most common are monopods, bipeds (e.g. humanoids), quadrupeds and hexapods, with one, two, four and six legs, respectively. Less common are robots with three, five, seven or more legs. There are two main types of leg designs: (a) Prismatic legs are characterised by an active or spring-loaded prismatic/linear joint, like Raibert's Hopping Machines [44]. (b) Articulated legs have a number of rotational/rotary joints, like all other examples shown in Figure 1. Each leg consists of links connected to each other with active and/or passive joints, also called Degrees of Freedom. While active joints are moved by an actuator, passive joints are often featuring a spring and/or damper. Exceptions are the so-called Passive Dynamic Walkers that use gravity and passive joints to walk down an inclined surface [35], and designs with a single actuator and linkages that create a walking motion (e.g. Theo Jansen's linkage used for the legs of his walking sculptures strandbeesten).

Legged robot designs are often inspired by nature to a certain degree, sometimes only calling leg joints with biological terms, or sometimes trying to mimic more precisely the kinematics and dynamic properties of humans and animals. The two principal leg configurations of multi-legged robots can be seen in Figure 1: cursorial/mammal type like IIT's HyQ [49], and sprawling/insect type like Tokyo Tech's Titan series [26]. The robots designed for dynamic gaits often feature springs or other elastic elements in the joints, legs or torso to temporarily store and release energy during periodic gaits (e.g. Raibert's Hopping Machines [44]). A common design rule is to keep the leg inertia low by reducing the weight and moving it close to the body.

The most common types of actuators used in today's robots are either electric, hydraulic or pneumatic. Electric actuators exist in different types: e.g. 


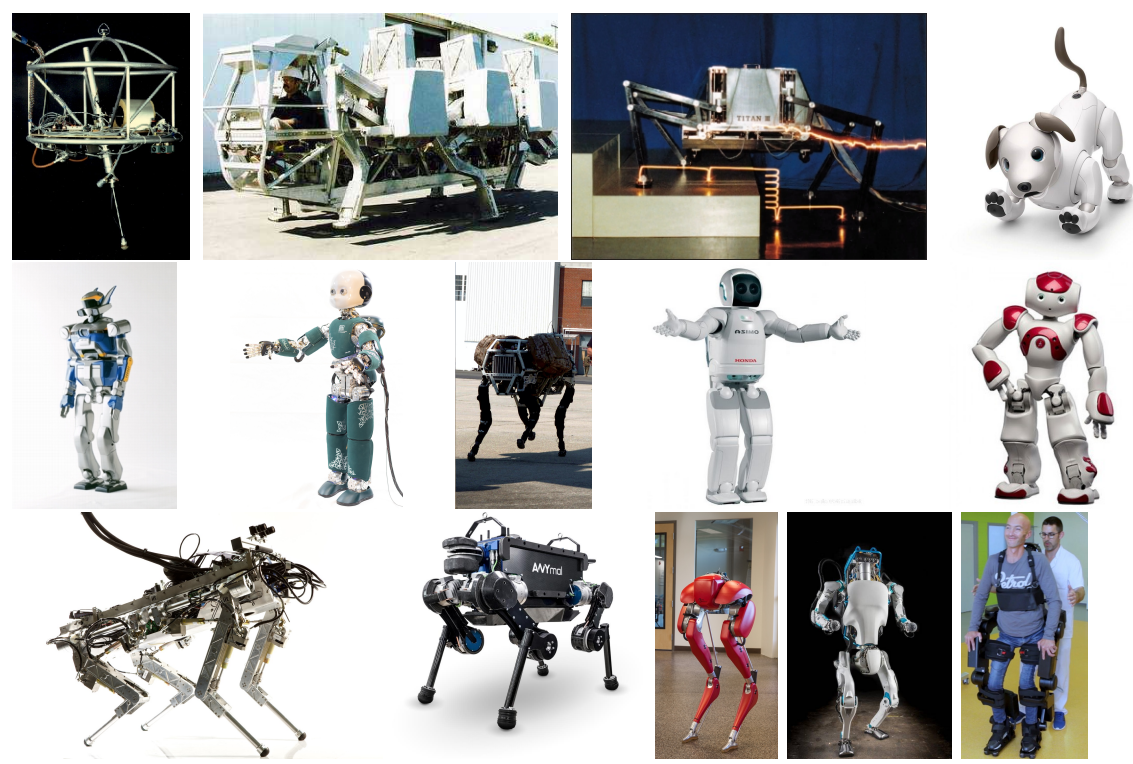

Fig. 1 Examples of legged robots. From left to right and top to bottom: Raibert's 3D hopper [44] (courtesy of Massachusetts Institute of Technology), Adaptive Suspension Vehicle [56] (courtesy of Ohio State University), Titan III [26] (courtesy of Tokyo Institute of Technology), AIBO [19] (courtesy of Sony Corp.), HRP-2 [38] (courtesy of Kawada Industries/AIST), iCub [53] (courtesy of Istituto Italiano di Tecnologia), BigDog [45] (courtesy of Boston Dynamics Inc./Softbank), ASIMO [52] (courtesy of Honda Corp.), NAO [22] (courtesy of Aldebaran/Softbank), HyQ [49] (courtesy of Istituto Italiano di Tecnologia), ANYmal [28] (courtesy of ANYbotics), Cassie [2] (courtesy of Agility Robotics), ATLAS [1] (courtesy of Boston Dynamics Inc./Softbank), self-balancing exo-skeleton [24] (courtesy of Wandercraft).

brushless, brushed, stepper, RC-servo motors. Examples of hydraulic actuators include cylinders, rotary vanes, axial pistons, rotary pistons. Pneumatic actuators exist as e.g. cylinders, McKibben muscles, expanding bladders. The hydraulic and pneumatic actuators need a source of pressurized fluid like oil or air, respectively. Non-traditional types of actuators for legged robots include electro-active polymers and shape memory alloys.

The correct choice of actuator type depends on the robot's field of application, requirements and operation environment. The advantages of electric actuators are: good power-to-weight ratio, availability in a wide range of sizes and prices, easy wire routing. Disadvantages are the need for sophisticated drive electronics and the need for reduction gears due to the generally low torque output. Gears have low impact resistance and might introduce backlash and friction. Low-gear-ratio drives with high-torque-output motors seem a promising direction (e.g. MIT's cheetah [58]). The pros of hydraulic actuators are: high power-to-weight ratio, high control bandwidth, impact resistance and easy heat removal from actuators. The cons are difficult hose 
routing across moving joints, limited commercial availability of small hydraulic components and thus high costs. Highly-integrated servo actuators with additive-manufactured metal bodies seem a promising direction (e.g. integrated servo actuators [4]). The pros of pneumatic actuators are: easy handling and availability of transmission fluid (e.g. air), high force-to-weight ratio (e.g. McKibben muscles). The cons are the low power-to-weight ratio, noise and difficult hose routing across moving joints. Nowadays, pneumatic actuators are rarely used for legged robots.

Proper actuator control is a crucial element in legged locomotion. For many years, stiff, position-controlled joints based on industrial manipulators dominated the field. While they are suitable for fast, repetitive, high-precision tasks in a well-known and structured environments, legged robots often have to deal with unstructured and unknown environments. Torque control on the other hand allows the implementation of different controllers (e.g. impedance control, passivity-based control, inverse dynamics) that are more suitable for locomotion.

\section{Dynamics of Legged Locomotion}

One of the major difficulties in making a legged robot walk or run is simply keeping its balance: where should the robot place its feet, how should it move its body in order to avoid falling and eventually reach its goal? This difficulty comes from the fact that contact forces with the environment are necessary to generate and control locomotion, but they are restricted by the mechanical laws of contact and the robot actuation limits.

The Newton equation of motion of the robot makes it clear that it needs external forces $f_{i}$ in order to move its Center of Mass $(\mathrm{CoM}) c$ in a direction other than that of gravity $g$ :

$$
m(\ddot{c}-g)=\sum_{i} f_{i},
$$

where $m$ is the total mass of the robot. And the Euler equation of motion makes it clear that the positions of the contact points $s_{i}$ with respect to the CoM $c$ are critical to keeping the angular momentum $L$ of the robot body around the CoM under control at the same time:

$$
\dot{L}=\sum_{i}\left(s_{i}-c\right) \times f_{i} .
$$

The problem is that in most cases, contacts are unilateral, meaning that the robot can push but not pull on the contact surfaces. Consequently, the forces $f_{i}$ can be oriented only in specific directions, further constrained by the limits of friction. These constraints can be accounted for by introducing the 
Center of Pressure (CoP) of the contact forces, also called the Zero Moment Point (ZMP) [48]. This characteristic point is bound to lie in a support area, delimited by the convex hull of contact points when the robot stands on flat ground, or obtained by projection of contact wrench constraints in more general contact situations [12].

Contact is usually considered completely rigid, disregarding visco-elastic deformations. This makes the situation binary: either there is contact and a contact force, or there is no contact and no contact force, which can be modeled as a complementarity condition [9]. When a leg collides with a surface, there is an impact and it is usually assumed that contact points will stick afterwards, though this can be undesirable [21]. Impacts and switching between different contact situations can be approached as a hybrid dynamical system, but this has limitations. The nonsmooth dynamics approach [9] can be more appropriate but it is usually disregarded because of its mathematical complexity.

How do we know if a legged robot is not going to fall? The condition for static balance is that the CoM must project vertically in a static-equilibrium polygon [8]. For dynamic motions, it is possible in theory to introduce the set of viable states, from which the robot is able to avoid falling [59]. It is typically intractable, however, to compute this set which is mostly conceptual, but simple sufficient conditions can be devised as follows.

Cyclic motions and equilibrium points are easy to identify as viable. And if the robot is able to reach such a cycle or equilibrium in a few steps, then it is viable as well [59]. In the other way, it has been shown for a simple bipedal model that if it is unable to reach such a cycle or equilibrium in two steps, then it is actually going to fall [62]. In conclusion, the capacity to reach a cycle or equilibrium in a few steps appears to be a good indicator of the capacity to avoid falling or not. This is the essence of the capturability analysis proposed in [43].

\section{Generation of walking and running motions}

Early legged robots relied on simple rules for generating walking and running motions, often inspired by biological hypotheses on animal motion control. Thanks to symmetries in bipedal or quadrupedal running gaits such as trot, pace and bound, the whole family of MIT LegLab's robots hopping on one, two or four legs, in 2D or 3D throughout the 1980s, could rely on the same control design [44]. The idea is to apply simple control laws independently to vertical oscillations, body orientation and foot placement, resulting in impressively robust and versatile locomotion. Note the focus on body orientation and foot placement, which relates directly to the Euler equation discussed above. 
Dominant theories on animal motion control include Central Pattern Generators (CPGs) and cascades of reflex motions which combine to generate the final motion. Van der Pol, Hopf or biologically inspired oscillators have been proposed as CPGs, generating quasi-cyclic motions in response to control signals such as locomotion speed or turning angle. Simple feedback loops ("reflexes") are then introduced to stabilize and adapt the motion of the robot, focusing again on body orientation and foot placement [46]. All the above concepts can be effectively combined with trunk stabilization techniques using proprioceptive [3] and exteroceptive feedback [54] in a modular way. Multi-legged locomotion poses the question of gait selection, which has been a continuous area of research for half a century now [36, 51]. The locomotion control concepts of multi-legged robots are largely the same as for bipeds. A crucial difference, however, is the much larger support area of multilegged robots that leads to an increased stability even under large motions of the CoM (e.g. arm or torso motions). Purely reflex based locomotion with no central coordination can be an option as well in quasi-static situations [10].

Alternatively, the mechanics of the robots can be tuned so that completely passive motions automatically land the feet on appropriate positions for balance, ending up with perfectly passive dynamic locomotion [35]. This inspired a very rich literature, including the Hybrid Zero Dynamics (HZD) approach [14] which generates robust cyclic walking motions in under-actuated systems such as biped robots with point feet. Promising robotic prostheses are also based on this approach [23], leveraging its automatic cycle synchronization.

Numerical optimization can be a critical tool to obtain efficient coordinations of limb motions, taking into account the complete nonlinear dynamics of the robots and objectives such as minimizing energy consumption. Current implementations are efficient, generic and reliable enough to generate online a wide range of motions [39]. Data-driven models can be handled by Reinforcement Learning (RL) approaches, potentially outperforming non data-driven options [29]. It is also possible to optimize simultaneously the motion and the mass distribution of the robot in order to maximize open-loop stability [37].

A pivotal observation is that only part of the motion of a legged robot is bound to contact forces. As seen in the Newton and Euler equations discussed above, contact forces $f_{i}$ relate to angular momentum $L$ and motion of the CoM $c$ with respect to contact points $s_{i}$. These are the elements of motion that need close supervision for balance. The proposition of artificial synergy synthesis [55] is to partition the generation of walking and running motion accordingly. It is the same observation that implicitly drives the Templates and Anchors approach and the long history of simple biomechanical models of legged locomotion that focus on a few meaningful degrees of freedom, mostly the motion of the CoM with respect to contact points, and abstract all the rest [20]. This approach has been tremendously fruitful for legged robots.

The role that the angular momentum $L$ has to play in locomotion and balance is still not entirely clear though. It is only indirectly related to the 
orientation of the main body because of nonholonomic effects, the same effects that allow cats to always fall back on their feet. If the main body does not rotate, the angular momentum $L$ is actually not zero during standard locomotion [60]. It is unclear then if it should be controlled to some specific value, and which one [57]. As a result, the most frequent option so far is to disregard angular momentum and regulate orientation instead [40].

Contact points $s_{i}$ are often planned beforehand, considering the environment of the robot and its goal, taking into account deterministically moving obstacles [13] through bounding boxes or swept volumes [41] on mildly rough terrain [63]. The corresponding CoM motion can be obtained then with a Model Predictive Control (MPC) scheme, imposing that the robot is always able to stop within a few steps in order to make sure that it remains constantly viable.

This approach has been successfully used in numerous biped and quadruped robots, including Kawada's HRP-2 [31], Honda's Asimo [52], Aldebaran's Nao [22], ANYbotics' ANYmal [6], IIT's HyQ [34], MIT's Cheetah [11]. Predefined steps can always be adapted if necessary, depending on the situation [15], what can prove crucial to walk over unstable terrain and sustain significant perturbations [17]. Efficient linear formulations have been possible by pre-defining the vertical motion of the CoM [32]. However, this leads to less efficient, less animal or human-like motion [7]. In very constrained and rough terrain, complex contact transitions can be required [25], but the resulting increase in computational load currently limits reactivity in such cases.

CoM, contact points and other aspects of robot motion for perception, manipulation and interaction usually involve Cartesian coordinates. Several whole-body motion control schemes have been proposed to control these different parts of the robot, such as standard inverse kinematics [18], Virtual Model Control [42], the Task Function approach [47], Operational Space Control [50]. Interestingly, these allow some form of decoupling between the different elements of the motion, further contributing to the artificial synergy synthesis approach discussed earlier.

\section{Applications}

The number of potential applications for legged robots is vast. Some of the most promising fields are: emergency response, inspection, maintenance, construction, security, logistics (e.g. curb to door) and elderly care. But currently limited functionality, insufficient robustness (hardware, software and control) and high cost have prevented a broad uptake by the market. One of the few successful application fields where several thousand copies have already been sold is edutainment (e.g. AIBO, NAO robots). Another one is research (e.g. 
study of legged locomotion of humans and animals, lower body rehabilitation).

\section{References}

1. ATLAS by Boston Dynamics Inc. https://www.bostondynamics.com/atlas. Last accessed on 15 February 2019

2. Cassie by Agility Robotics, http://www.agilityrobotics.com/robots/. Last accessed on 15 February 2019

3. Barasuol, V., Buchli, J., Semini, C., Frigerio, M., De Pieri, E.R., Caldwell, D.G.: A reactive controller framework for quadrupedal locomotion on challenging terrain. pp. 2554-2561 (2013). DOI 10.1109/ICRA.2013.6630926

4. Barasuol, V., Villarreal-Magaña, O.A., Sangiah, D., Frigerio, M., Baker, M., Morgan, R., Medrano-Cerda, G.A., Caldwell, D.G., Semini, C.: Highly-integrated hydraulic smart actuators and smart manifolds for high-bandwidth force control. Frontiers in Robotics and AI 5, 51 (2018). DOI 10.3389/frobt.2018.00051

5. Bares, J., Wettergreen, D.: Dante II: technical description, results and lessons learned. International Journal of Robotics Research 18(7), 621-649 (1999)

6. Bellicoso, C.D., Jenelten, F., Gehring, C., Hutter, M.: Dynamic locomotion through online nonlinear motion optimization for quadrupedal robots. IEEE Robotics and Automation Letters 3(3), 2261-2268 (2018)

7. Brasseur, C., Sherikov, A., Collette, C., Dimitrov, D., Wieber, P.B.: A robust linear MPC approach to online generation of $3 \mathrm{D}$ biped walking motion. In: Proceedings of the IEEE-RAS International Conference on Humanoid Robots (2015)

8. Bretl, T., Lall, S.: Testing static equilibrium for legged robots. IEEE Transactions on Robotics 24(4), 794-807 (2008)

9. Brogliato, B.: Nonsmooth Mechanics. Communications and Control Engineering Series, Springer-Verlag, London (1999)

10. Brooks, R.: A robot that walks; emergent behaviors from a carefully evolved network. In: Proceedings of the IEEE International Conference on Robotics \& Automation, pp. 292-296 (1989)

11. Carlo, J.D., Wensing, P.M., Katz, B., Bledt, G., , Kim, S.: Dynamic locomotion in the MIT Cheetah 3 through convex Model Predictive Control. In: Proceedings of the IEEE/RSJ International Conference on Intelligent Robots \& Systems (2018)

12. Caron, S., Pham, Q.C., Nakamura, Y.: ZMP support areas for multi-contact mobility under frictional constraints. IEEE Transactions on Robotics 33(1), 67-80 (2017)

13. Chestnutt, J., Michel, P., Kuffner, J., Kanade, T.: Locomotion among dynamic obstacles for the Honda Asimo. In: Proceedings of the IEEE/RSJ International Conference on Intelligent Robots \& Systems (2007)

14. Chevallereau, C., Abba, G., Aoustin, Y., Plestan, F., Westervelt, E., Canudas de Wit, C., Grizzle, J.: RABBIT: A testbed for advanced control theory. IEEE Control Systems Magazine 23(5), 57-79 (2003)

15. Diedam, H., Dimitrov, D., Wieber, P.B., Mombaur, K., Diehl, M.: Online walking gait generation with adaptive foot positioning through linear model predictive control. In: Proceedings of the IEEE/RSJ International Conference on Intelligent Robots \& Systems (2008)

16. Endo, G., Hirose, S.: Study on roller-walker (system integration and basic experiments). In: Proceedings of the IEEE International Conference on Robotics \& Automation, pp. 2032-2037 (1999)

17. Feng, S., Xinjilefu, X., Atkeson, C.G., Kim, J.: Robust dynamic walking using online foot step optimization. In: Proceedings of the IEEE/RSJ International Conference on Intelligent Robots \& Systems (2016) 
18. Fujimoto, Y., Kawamura, A.: Proposal of biped walking control based on robust hybrid position/force control. In: Proceedings of the IEEE International Conference on Robotics \& Automation, pp. 2724-2730 (1996)

19. Fujita, M., Kitano, H.: Development of an autonomous quadruped robot for robot entertainment. Autonomous Robots 5, 7-18 (1998)

20. Full, R.J., Koditschek, D.E.: Templates and anchors: Neuromechanical hypotheses of legged locomotion on land. Journal of Experimental Biology 202, 3325-3332 (1999)

21. Gamus, B., Or, Y.: Analysis of dynamic bipedal robot locomotion with stick-slip transitions. In: Submitted to the IEEE International Conference on Robotics \& Automation (2013)

22. Gouaillier, D., Collette, C., Kilner, C.: Omni-directional closed-loop walk for NAO. In: Proceedings of the IEEE-RAS International Conference on Humanoid Robots (2010)

23. Gregg, R.D., Lenzi, T., Hargrove, L.J., Sensinger, J.W.: Virtual constraint control of a powered prosthetic leg: From simulation to experiments with transfemoral amputees. IEEE Transactions on Robotics 30(6), 1455-1471 (2014)

24. Gurriet, T., Finet, S., Boeris, G., Duburcq, A., Hereid, A., Harib, O., Masselin, M., Grizzle, J., Ames, A.D.: Towards restoring locomotion for paraplegics: Realizing dynamically stable walking on exoskeletons. In: Proceedings of the IEEE International Conference on Robotics \& Automation (2018)

25. Hauser, K., Bretl, T., Latombe, J.C., Harada, K., Wilcox, B.: Motion planning for legged robots on varied terrain. International Journal of Robotics Research (2008)

26. Hirose, S., Fukuda, Y., Yoneda, K., Nagakubo, A., Tsukagoshi, H., Arikawa, K., Endo, G., Doi, T., Hodoshima, R.: Quadruped walking robots at tokyo institute of technology. IEEE Robotics and Automation Magazine 16, 104-114 (2009)

27. Hirose, S., Nagakubo, A., Toyama, R.: Machine that can walk and climb on floors, walls and ceilings. In: Proceedings of the International Conference on Advanced Robotics, pp. $753-758$ (1991)

28. Hutter, M., Gehring, C., Lauber, A., Gunther, F., Bellicoso, C.D., Tsounis, V., Fankhauser, P., Diethelm, R., Bachmann, S., Bloesch, M., Kolvenbach, H., Bjelonic, M., Isler, L., Meyer, K.: Anymal - toward legged robots for harsh environments. Advanced Robotics 31(17), 918-931 (2017)

29. Hwangbo, J., Lee, J., Dosovitskiy, A., Bellicoso, D., Tsounis, V., Koltun, V., Hutter, M.: Learning agile and dynamic motor skills for legged robots. Science Robotics 4(26) (2019)

30. Iagnemma, K., Dubowsky, S.: Traction control of wheeled robotic vehicles in rough terrain with application to planetary rovers. International Journal of Robotics Research 23(10-11), 1029-1040 (2004)

31. Kajita, S., Kanehiro, F., Kaneko, K., Fujiwara, K., Harada, K., Yokoi, K., Hirukawa, H.: Biped walking pattern generation by using preview control of Zero Moment Point. In: Proceedings of the IEEE International Conference on Robotics \& Automation, pp. 1620-1626 (2003)

32. Kajita, S., Tani, K.: Study of dynamic biped locomotion on rugged terrain - derivation and application of the linear inverted pendulum mode -. In: Proceedings of the IEEE International Conference on Robotics \& Automation, pp. 1405-1411 (1991)

33. Kim, S., Asbeck, A., Provancher, W., Cutkosky, M.R.: Spinybotii: Climbing hard walls with compliant microspines. In: Proceedings of the International Conference on Advanced Robotics, pp. 18-20 (2005)

34. Mastalli, C., Focchi, M., Havoutis, I., Radulescu, A., Calinon, S., Buchli, J., Caldwell, D.G., Semini, C.: Trajectory and foothold optimization using low-dimensional models for rough terrain locomotion. In: Proceedings of the IEEE International Conference on Robotics \& Automation (2017)

35. McGeer, T.: Passive Dynamic Walking. Simon Fraser University Technical Report (1988)

36. McGhee, R.B., Frank, A.A.: On the stability properties of quadruped creeping gaits. Mathematical Biosciences 3, 331 - 351 (1968) 
37. Mombaur, K.: Using optimization to create self-stable human-like running. Robotica 27(3), 321-330 (2009)

38. Morisawa, M., Harada, K., Kajita, S., Kaneko, K., Kanehiro, F., Fujiwara, K., Nakaoka, S., Hirukawa, H.: A biped pattern generation allowing immediate modification of foot placement in real-time. In: Proceedings of the IEEE-RAS International Conference on Humanoid Robots (2006)

39. Neunert, M., Stäuble, M., Giftthaler, M., Bellicoso, C.D., Carius, J., Gehring, C., Hutter, M., Buchli, J.: Whole-body nonlinear model predictive control through contacts for quadrupeds. IEEE Robotics and Automation Letters 3(3), 1458-1465 (2018)

40. Ott, C., Roa, M.A., Hirzinger, G.: Posture and balance control for biped robots based on contact force optimization. In: Proceedings of the IEEE-RAS International Conference on Humanoid Robots (2011)

41. Perrin, N., Stasse, O., Baudoin, L., Lamiraux, F., Yoshida, E.: Fast humanoid robot collision-free footstep planning using swept volume approximations. IEEE Transactions on Robotics 28(2), 427-439 (2012)

42. Pratt, J., Chew, C.M., Torres, A., Dilworth, P., Pratt, G.: Virtual model control: An intuitive approach for bipedal locomotion. International Journal of Robotics Research 20, 129-143 (2001)

43. Pratt, J., Tedrake, R.: Velocity based stability margins for fast bipedal walking. In: Proceedings of the Ruperto Carola Symposium on Fast Motion in Biomechanics and Robotics (2005)

44. Raibert, M.: Legged Robots that Balance. MIT Press, Cambridge (1986)

45. Raibert, M., Blankespoor, K., Nelson, G., Playter, R., the BigDog Team: Bigdog, the rough-terrain quadruped robot. In: Proceedings of the 17th World Congress The International Federation of Automatic Control (IFAC) (2008)

46. Righetti, L., Ijspeert, A.: Programmable Central Pattern Generators: an application to biped locomotion control. In: Proceedings of the IEEE International Conference on Robotics \& Automation (2006)

47. Saab, L., Ramos, O.E., Keith, F., Mansard, N., Souères, P., Fourquet, J.Y.: Dynamic whole-body motion generation under rigid contacts and other unilateral constraints. IEEE Transactions on Robotics 29(2), 346-362 (2013)

48. Sardain, P., Bessonnet, G.: Forces acting on a biped robot. Center of Pressure-Zero Moment Point. IEEE Transactions on Systems, Man, and Cybernetics - Part A 34(5), 630-637 (2004)

49. Semini, C., Tsagarakis, N.G., Guglielmino, E., Focchi, M., Cannella, F., Caldwell, D.G.: Design of HyQ - a hydraulically and electrically actuated quadruped robot. Journal of Systems and Control Engineering 225(6), 831-849 (2011)

50. Sentis, L., Park, J., Khatib, O.: Compliant control of multicontact and center-of-mass behaviors in humanoid robots. IEEE Transactions on Robotics 26(3), 483-501 (2010)

51. Smit-Anseeuw, N., Gleason, R., Vasudevan, R., Remy, C.D.: The energetic benefit of robotic gait selection - a case study on the robot RAMone. IEEE Robotics and Automation Letters 2(2), 1124-1131 (2017)

52. Takenaka, T., Matsumoto, T., Yoshiike, T.: Real time motion generation and control for biped robot -1st report: Walking gait pattern generation-. In: Proceedings of the IEEE/RSJ International Conference on Intelligent Robots \& Systems (2009)

53. Tsagarakis, N., Metta, G., Sandini, G., Vernon, D., Beira, R., Becchi, F., Righetti, L., Santos-Victor, J., Ijspeert, A., Carrozza, M., Caldwell, D.: icub - the design and realization of an open humanoid platform for cognitive and neuroscience research. Journal of Advanced Robotics, Special Issue on Robotic platforms for Research in Neuroscience pp. 1151-1175 (2007)

54. Villarreal, O., Barasuol, V., Camurri, M., Focchi, M., Franceschi, L., Pontil, M., Caldwell, D.G., Semini, C.: Fast and continuous foothold adaptation for dynamic locomotion through convolutional neural networks. IEEE Robotics and Automation Letters (RA-L) (2019) 
55. Vukobratović, M.K.: Contribution to the study of anthropomorphic systems. Kybernetika 8(5), 404-418 (1972)

56. Waldron, K.J., McGhee, R.B.: The adaptive suspension vehicle. IEEE Control Systems Magazine 6, 7-12 (1986)

57. Wensing, P., Orin, D.: Improved computation of the humanoid centroidal dynamics and application for whole-body control. International Journal of Humanoid Robotics 13(01), 1550,039 (2016)

58. Wensing, P., Wang, A., Seok, S., Otten, D., Lang, J., Kim, S.: Proprioceptive actuator design in the MIT Cheetah: Impact mitigation and high-bandwidth physical interaction for dynamic legged robots. IEEE Transactions on Robotics 33(3), 509-522 (2017)

59. Wieber, P.B.: On the stability of walking systems. In: Proceedings of the International Workshop on Humanoids and Human Friendly Robots (2002)

60. Wieber, P.B.: Holonomy and nonholonomy in the dynamics of articulated motion. In: Proceedings of the Ruperto Carola Symposium on Fast Motion in Biomechanics and Robotics (2005)

61. Yano, T., Numao, S., Kitamura, Y.: Development of a self-contained wall climbing robot with scanning type suction cups. In: Proceedings of the IEEE/RSJ International Conference on Intelligent Robots \& Systems, pp. 249-254 (1998)

62. Zaytsev, P., Hasaneini, S.J., Ruina, A.: Two steps is enough: No need to plan far ahead for walking balance. In: Proceedings of the IEEE International Conference on Robotics \& Automation (2015)

63. Zucker, M., Bagnell, J.A., Atkeson, C.G., Kuffner, J.: An optimization approach to rough terrain locomotion. In: Proceedings of the IEEE International Conference on Robotics \& Automation (2010) 